\title{
The secreted protein acidic and rich in cysteine is a critical mediator of cell death program induced by WIN/TRAIL combined treatment in osteosarcoma cells
}

\author{
ANTONIETTA NOTARO $^{1}$, SELENIA SABELLA ${ }^{1}$, ORNELLA PELLERITO ${ }^{2}$, \\ RENZA VENTO $^{1}$, GIUSEPPE CALVARUSO ${ }^{1}$ and MICHELA GIULIANO ${ }^{1}$ \\ ${ }^{1}$ Department of Biological, Chemical and Pharmaceutical Sciences and Technologies, \\ Laboratory of Biochemistry, University of Palermo, Polyclinic, Palermo 90128, Italy; \\ ${ }^{2}$ Department of Genetics and Development, Toronto Western Hospital, Toronto, ON M5T 2S8, Canada
}

Received September 28, 2015; Accepted November 5, 2015

DOI: 10.3892/ijo.2015.3307

\begin{abstract}
Secreted protein acidic and rich in cysteine (SPARC) is a multi-functional protein which modulates cell-cell and cell-matrix interactions. In cancer cells, SPARC behaves as a tumor promoter in a number of tumors, but it can also act as a tumor suppressor factor. Our previous results showed that the synthetic cannabinoid WIN55,212-2 (WIN), a potent cannabinoid receptor agonist, is able to sensitize osteosarcoma MG63 cells to TNF-related apoptosis-inducing ligand (TRAIL)-induced apoptosis which is accompanied with endoplasmic reticulum (ER)-stress induction and the increase in autophagic markers. In the present investigation, we studied the role of SPARC in WIN/TRAIL-induced apoptosis demonstrating that WIN increased the level of SPARC protein and mRNA in a time-dependent manner. This event was functional to WIN/TRAIL-dependent apoptosis as demonstrated by RNA interfering analysis which indicated that SPARC-silenced cells were less sensitive to cytotoxic effects induced by the combined treatment. Our experiments also demonstrate that SPARC interacts with caspase- 8 thus probably favoring its translocation to plasma membrane and the activation of extrinsic apoptotic pathway. In conclusion, to the best of our knowledge, our results are the first to show that WIN-dependent increase in the level of SPARC plays a critical role in sensitizing osteosarcoma cells to TRAIL action.
\end{abstract}

\section{Introduction}

Osteosarcoma is the most common form of primary bone malignancy, which occurs predominantly in infants and

Correspondence to: Professor Michela Giuliano, Department of Biological, Chemical and Pharmaceutical Sciences and Technologies, Laboratory of Biochemistry, University of Palermo, Policlinic, Via del Vespro 129, Palermo 90128, Italy

E-mail: michela.giuliano@unipa.it

Key words: SPARC, cannabinoids, osteosarcoma, apoptosis, caspase-8 activation adolescents (1). It represents the prevalent cause of cancerrelated death for children with an incidence of 4-5 cases $/ 10^{8}$. Patients with osteosarcoma are routinely treated by combining surgery and high-dose chemotherapy and this has significantly improved the 5-year survival rate over time. Nevertheless, osteosarcoma shows high propensity to metastasize and invade surrounding tissue, the lung being the most common site of initial metastatic disease or, less frequently, the other bones (2). Therefore, it is important to identify novel therapeutic strategies which can improve the general conditions and the overall survival rate of patients with osteosarcoma.

SPARC (secreted protein acidic and rich in cysteine) also called Osteonectin or BM-40, is a non-structural matricellular glycoprotein expressed in a variety of mammalian tissues $(3,4)$. The effects of SPARC on cell behaviour are highly tissue specific and concern modification of cell shape, migration, proliferation, differentiation and survival (5-9). SPARC is also known to modulate cell-cell and cell-matrix interactions, and to influence de-adhesive and cell growth regulatory properties (10). Moreover, its expression is related to the ability to regulate processes such as bone formation, fibrosis and tissue repair (11). SPARC is differentially expressed in various tumors including breast and colorectal cancers, melanoma or glioma (12-17). Many studies show that in cancer cells SPARC modulates proliferation, apoptosis, invasion and angiogenesis. Its overexpression can promote tumor vascularization by interacting with cytokines, such as VEGF and PDGF and stimulating the secretion of metalloproteases (18). This could lead to the conclusion that SPARC is a tumor promoter protein with pro-invasive activity. However, the role of SPARC in tumorigenesis is more complex and seems to be cell-type specific owing to its diverse functions in a given micro-environment (10). Indeed, in other tumors, such as ovarian and gastric cancer cells, SPARC is significantly downregulated and its restoring mediates the inhibition of cell proliferation $(19,20)$.

Recently, we have demonstrated that osteosarcoma MG63 cells are sensitive to the synthetic cannabinoid WIN55,212-2, an agonist of cannabinoid receptors (21). In these cells WIN is able to increase the level of the main markers of endoplasmic 
reticulum (ER) stress and mediate the overexpression of tumor suppressor factor PAR-4. The consequence of these effects is the sensitization to the action of the cytokine TRAIL and the induction of apoptotic cell death. In the present study, we aimed to elucidate the role of SPARC in the effects induced by WIN in osteosarcoma MG63 cells.

\section{Materials and methods}

Cell cultures. Human osteosarcoma MG63 cells were acquired from Interlab Cell Line Collection (ICLC; Genova, Italy). Cells were cultured at $37^{\circ} \mathrm{C}$ in Dulbecco's modified Eagle's medium (DMEM), supplemented with $10 \%(\mathrm{v} / \mathrm{v})$ heat-inactivated fetal bovine serum (FBS), $2.0 \mathrm{mM} \mathrm{L}$-glutamine, and antibiotic antimycotic solution ( $100 \mathrm{U} / \mathrm{ml}$ penicillin, $100 \mu \mathrm{g} / \mathrm{ml}$ streptomycin and $250 \mathrm{ng} / \mathrm{ml}$ amphotericin B; Sigma) in a humidified atmosphere containing $5 \% \mathrm{CO}_{2}$. For the experiments, cells were seeded at $60-70 \%$ confluence. After overnight incubation, culture medium was replaced with fresh DMEM containing low percentage of FBS (2\%) and cells were treated with the cannabinoid WIN55,212-2 (WIN) (Sigma Aldrich S.R.L., Milan, Italy). Control cells were cultured in the presence of vehicle alone (DMSO).

Cell viability assay. Cell viability was determined by 3-[4,5-dimethylthiazolyl-2]2,5-diphenyl-tetrazolium bromide (MTT; Sigma Aldrich S.R.L.) assay as previously reported (22).

Western blot analysis. Protein extracts were prepared by washing the cells in phosphate-buffer saline (PBS) and incubating for $20 \mathrm{~min}$ in ice-cold lysis buffer supplemented with protease inhibitor cocktail, as previously reported (23). After sonication three times for $10 \mathrm{sec}$, proteins were quantified by Bradford method and equal amounts $(40 \mu \mathrm{g})$ were separated by SDS-PAGE and then electrotransferred to a nitrocellulose membrane for the detection with specific antibodies. The blots were developed using the alkaline phosphatase colorimetric or enhanced chemiluminescence (ECL) labeling systems. Optical densities of the bands were analyzed with Quantity One Imaging software from Bio-Rad Laboratories. The correct protein loading was verified by means of both red Ponceau staining and immunoblotting for actin. The results shown in the figures are representative of four independent experiments with similar results.

Semi-quantitative RT-PCR analysis for SPARC. RNA was isolated using RNeasy mini kit (Qiagen, Milan, Italy). cDNA was amplified from $1 \mu \mathrm{g}$ of RNA by using QuantiTect reverse transcription kit (Qiagen) followed by polymerase chain reaction (PCR). The reactions omitting reverse transcriptase enzyme served as negative control. GAPDH was used as a housekeeping gene to demonstrate equal loading of RNA.

The amplified products were resolved by agarose gel electrophoresis (1\% agarose, $0.5 \mu \mathrm{g} / \mathrm{ml}$ ethidium bromide; Sigma-Aldrich), and the bands were visualized and photographed with ChemiDoc XRS (Bio-Rad Laboratories Srl, Milan, Italy). The primer sequences (Proligo USA, Milan, Italy) are as follows: SPARC, forward 5'-TGATGATGGTGC AGAGGAAA-3' and reverse 5'-GGGGGATGTATTTGCAA GG-3'; GAPDH, forward 5'-TGACATCAAGAAGGTGA-3'

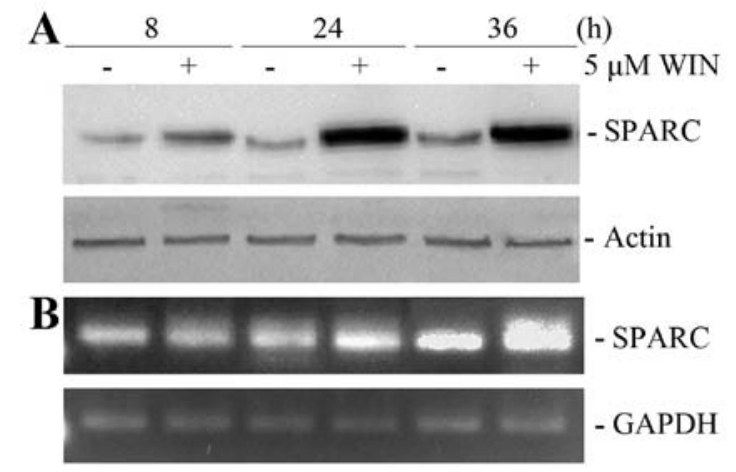

Figure 1. WIN induces a marked increase in SPARC expression in osteosarcoma MG63 cells. (A) Time-dependent effect induced by $5 \mu \mathrm{M}$ WIN on the level of SPARC. The results were obtained by immunoblotting employing a specific antibody as reported in Materials and methods. Actin blot was included as a loading control. (B) Semi-quantitative RT-PCR analysis of SPARC expression in MG63 cells. After treatment with $5 \mu \mathrm{M}$ WIN, total cellular RNA was extracted and RT-PCR was performed as described in Materials and methods. GAPDH mRNA levels were evaluated as an internal control.

and reverse 5'-TCCACCACCCTGTTGCTGTA-3'. For PCR analysis, the following protocol was performed: initial denaturation at $94^{\circ} \mathrm{C}$ for $2 \mathrm{~min}$; denaturation at $94^{\circ} \mathrm{C}$ for $15 \mathrm{sec}$, annealing at $60^{\circ} \mathrm{C}$ for $30 \mathrm{sec}$ and extension at $72^{\circ} \mathrm{C}$ for $30 \mathrm{sec}$ for 35 cycles and a final extension at $72^{\circ} \mathrm{C}$ for $10 \mathrm{~min}$.

Gene silencing using siRNAs. Small interfering RNAs (siRNAs) against SPARC (5'-AACAAGACCUU CGACUCUU CC-3') (siSPARC), CHOP (D-004819-01-0005 and D-00481902-0005) (siCHOP), and scrambled siRNA (siScr), as a negative non-silencing control, were purchased from Dharmacon RNA Technologies (Chicago, IL, USA) and GRP78 (siGRP78) from Santa Cruz Biotechnology (Santa Cruz, CA, USA).

Cells $\left(10^{5}\right)$ were seeded in 6-well plates and cultured in antibiotic-free DMEM supplemented with $2.0 \mathrm{mM}$ L-glutamine, until $\sim 50 \%$ confluence. Then, cells were transfected with $30 \mathrm{nM}$ siSPARC in the presence of $5 \mu 1$ Metafectene Pro (Biontex Laboratories GmbH, Martinsried/Planegg, Germany), with $100 \mathrm{nM}$ siCHOP in the presence of $6.2 \mu \mathrm{l}$ of Metafectene Pro or with $50 \mathrm{nM}$ siGRP78 in the presence of $4 \mu 1$ Lipofectamine 2000 (Invitrogen Life Technologies, Monza, Italy) in a final volume of $1 \mathrm{ml}$ serum-free medium. The reaction was stopped after $6 \mathrm{~h}$ replacing the culture medium with fresh DMEM + $10 \%$ FBS. After $24 \mathrm{~h}$ from transfection, silenced cells were treated with WIN for another $24 \mathrm{~h}$. Only for SPARC silencing, cells were treated after $48 \mathrm{~h}$ from transfection.

Caspase-8/SPARC coimmunoprecipitation. The caspase-8/ SPARC complex was detected in MG63 cells by immunoprecipitation either with the anti-caspase- 8 or the anti-SPARC antibodies. Cell lysates were subjected to centrifugation at $14,000 \mathrm{rpm}$ at $4^{\circ} \mathrm{C}$ for $15 \mathrm{~min}$. Proteins concentration was evaluated by using Bradford assay and then was adjusted to $500 \mu \mathrm{g}$ of protein in a final volume of $500 \mu \mathrm{l}$ of RIPA buffer. After pre-clearing phase by $1 \mathrm{~h}$ of incubation at room temperature with $20 \mu \mathrm{l}$ of protein A/G Plus-agarose beads (Santa Cruz Biotechnology), lysates were spun at 14,000 rpm for $10 \mathrm{~min}$. 
A

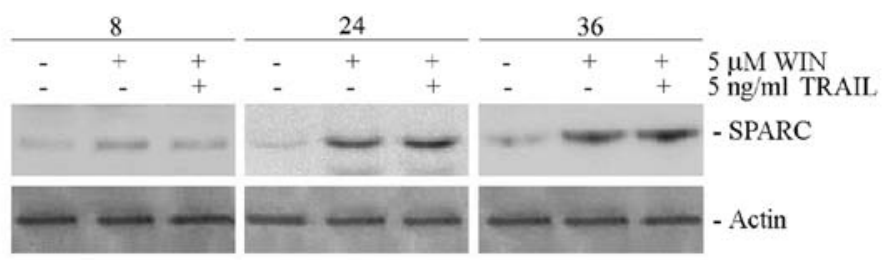

B

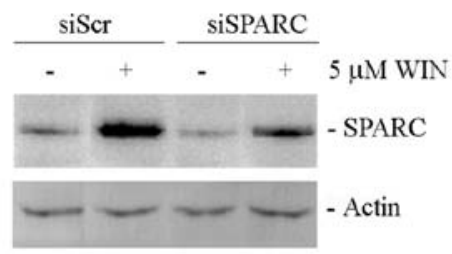

C

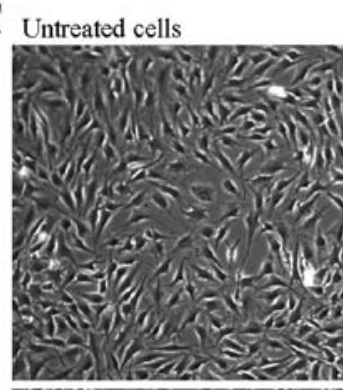

$5 \mathrm{ng} / \mathrm{ml}$ TRAII
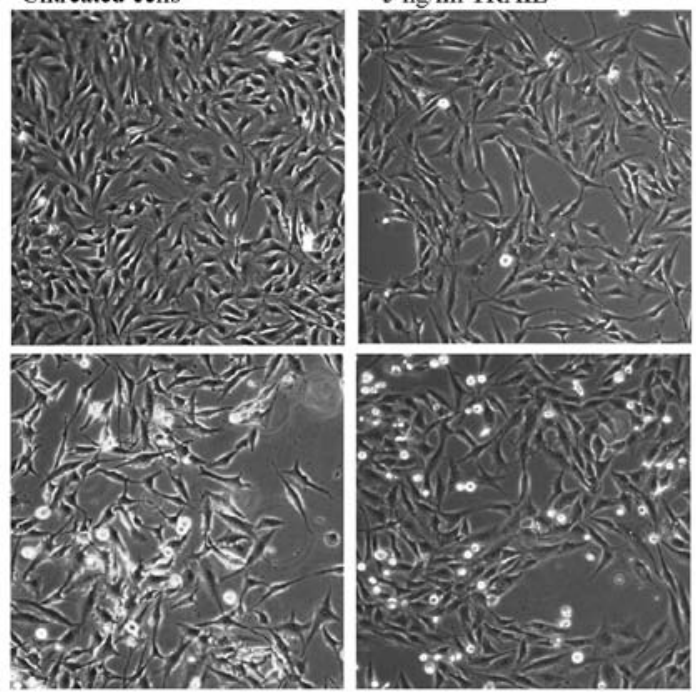

\section{D}

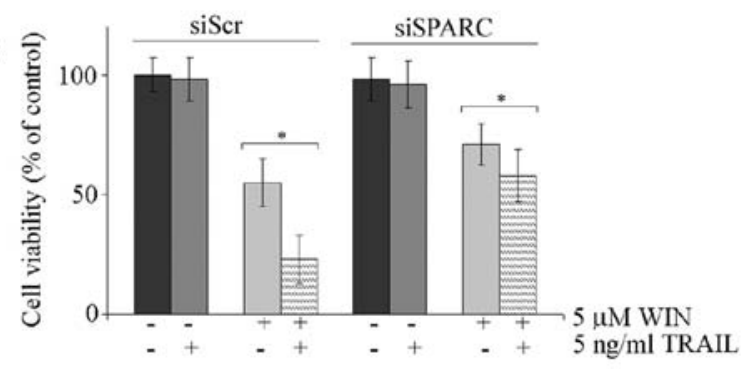

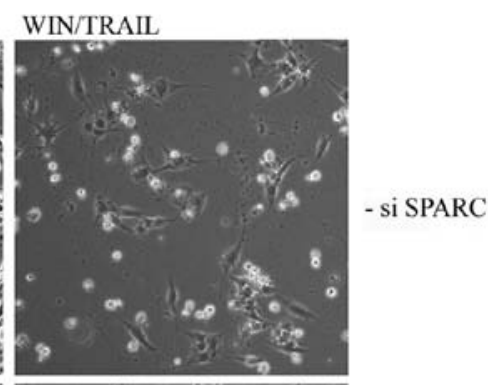

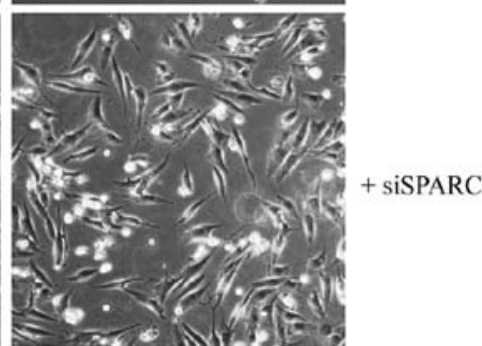

Figure 2. SPARC upregulation is functional in WIN-induced sensitization to TRAIL. (A) Time-dependent effect induced by $5 \mu \mathrm{M}$ WIN and/or 5 ng/ml TRAIL on SPARC level. The results were obtained by immunoblotting employing a specific antibody as reported in Materials and methods. Actin blot was included as a loading control. (B) Effect of SPARC silencing on the level of the protein. SPARC silencing was carried out as reported in Materials and methods. After $48 \mathrm{~h}$ from the transfection, cells were incubated in fresh medium for $24 \mathrm{~h}$ in the presence of $5 \mu \mathrm{M}$ WIN. Finally, immunoblotting analysis was performed to evaluate the expression levels of SPARC. Actin blot was included as a loading control. (C) Morphological effects induced by WIN and/or TRAIL treatment for $24 \mathrm{~h}$ in siSPARC silenced or unsilenced (siScr) cells (original magnification, x200). (D) Effects of $5 \mu \mathrm{M}$ WIN employed alone or in combination with $5 \mathrm{ng} / \mathrm{ml} \mathrm{TRAIL}$ on siSPARC or siScr silenced MG63 cell viability. Cell survival was estimated by MTT assay after $24 \mathrm{~h}$ of treatment, as reported in Materials and methods, and expressed as the percentage of control cells. Data are the means \pm SE of four independent experiments involving triplicate assays. ${ }^{*}<0.01$ vs. untreated cells.

Anti-SPARC or anti-caspase- 8 antibodies or the appropriate antibodies used as negative controls were added to the supernatant and incubated overnight at $4^{\circ} \mathrm{C}$, followed by immunoprecipitation with protein $\mathrm{A} / \mathrm{G}$ Plus-agarose beads for $2 \mathrm{~h}$ of incubation at room temperature. The beads were washed three times with PBS and the bound proteins were detached by boiling the beads in the presence of sample buffer for $5 \mathrm{~min}$. The supernatants were subjected to electrophoresis on SDS-polyacrylamide gels and then electroblotted on nitrocellulose filter for the detection of the immunoprecipitate complex.

Statistical analysis. Cell viability data were expressed as the mean \pm SE and evaluated by the Student's t-test. Differences were considered to indicate a statistically significant result when the P-value was $<0.01$.

\section{Results}

WIN induces overexpression of SPARC protein. In a previous study we demonstrated that WIN, a synthetic ligand of cannabinoid receptors, is able to sensitize osteosarcoma MG63 and Saos-2 cells to TRAIL action (21). In the present study we first analysed the effect of WIN on modulating the level of SPARC, a factor which plays different roles on cell proliferation and migration. As shown in Fig. 1A, $5 \mu \mathrm{M}$ WIN, a concentration which is responsible for the morphological and TRAIL-sensitizing effects, induced a time-dependent increase in the level of SPARC in MG63 cells. The effect was already evident after $8 \mathrm{~h}$ of treatment and reached the maximum at 24-36 h. Similar results were obtained using semi-quantitative RT-PCR, thus, demonstrating that the increase in SPARC protein was associated with transcriptional activation. 

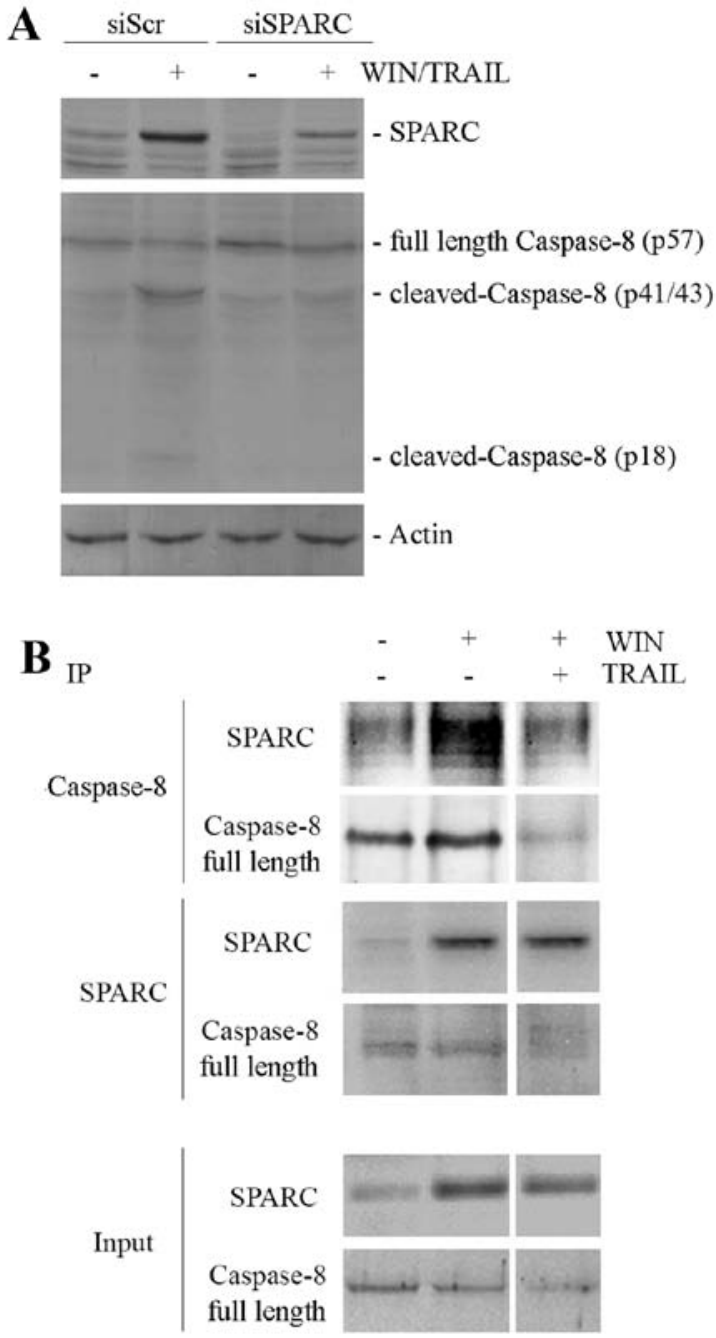

Figure 3. SPARC interacts with caspase-8. (A) Effects of anti-SPARC siRNA on caspase-8 level in WIN/TRAIL-treated MG63 cells. Cells were transfected as reported in Materials and methods. After $48 \mathrm{~h}$ from the transfection, cells were incubated in fresh medium for other $24 \mathrm{~h}$ in the presence of $5 \mu \mathrm{M}$ WIN and $5 \mathrm{ng} / \mathrm{ml}$ TRAIL. Immunoblotting analysis was performed to evaluate the expression levels of proteins. Actin blot was included as a loading control. (B) Co-localization of SPARC and caspase- 8 determined by co-IP studies after exposure for $24 \mathrm{~h}$ to $5 \mu \mathrm{M}$ WIN employed alone or in combination with $5 \mathrm{ng} / \mathrm{ml}$ TRAIL. Briefly, $500 \mu \mathrm{g}$ of protein was immunoprecipitated with antibodies against caspase-8 (C-terminus) and SPARC, and immunoblotted for SPARC or caspase-8. The lanes of Input represent the western blot analysis of starting material before immunoprecipitation.

SPARC is responsible for TRAIL-sensitization via the interaction with caspase- 8 protease. We were interested to clarify whether SPARC can be a critical mediator of the cytotoxic action induced by WIN/TRAIL combined treatment. After confirming that the level of SPARC remained high also in the presence of WIN/TRAIL combined treatment (Fig. 2A), we analysed the effect of the downregulation of SPARC expression by a specific siRNA which knocked down the endogenous protein level by $\sim 70 \%$ (Fig. 2B). In SPARC silenced cells, WIN/TRAIL combined treatment exerted smaller effects than that observed in non-silenced cells. After $24 \mathrm{~h}$ of treatment with WIN or WIN/TRAIL combination, siSPARC-transfected cells showed a morphology, which was very similar to that observed in control cells (Fig. 2C). Moreover, MTT assay evidenced that in siSPARC-transfected cells the reduction in

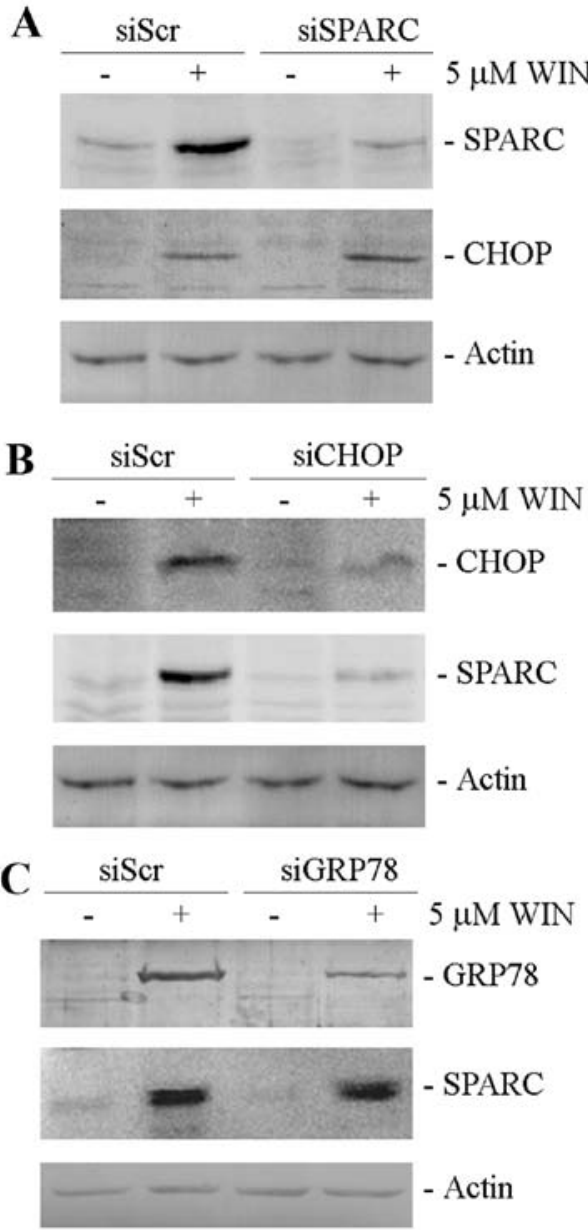

Figure 4. SPARC increase is related to WIN-induced ER stress. Effects of (A) anti-SPARC, (B) anti-CHOP or (C) anti-GRP78 siRNAs on SPARC or CHOP expression in WIN-treated MG63 cells. Cells were transfected for $6 \mathrm{~h}$ with specific siRNAs or with scrambled siRNA (siScr) as reported in Materials and methods. After $24 \mathrm{~h}$ from the transfection (or $48 \mathrm{~h}$ for SPARC), cells were incubated in fresh medium for another $24 \mathrm{~h}$ in the presence of $5 \mu \mathrm{M}$ WIN. Immunoblotting analysis was performed to evaluate the expression levels of CHOP, SPARC and GRP78. Actin blots were included as a loading control.

MG63 cell viability induced by WIN/TRAIL treatment was approximately $40 \mathrm{vs} .80 \%$ of that observed in siSicr-transfected cells exposed to the same treatment (Fig. 2D).

Caspase- 8 is the main initiating protease activity in extrinsic apoptotic pathway and we previously demonstrated that its activation is a very probable event responsible for the WIN/TRAIL-dependent cytotoxic effect (21). Moreover, as recently reported, SPARC can potentiate the apoptotic pathway by enhancing the signalling cascade in a caspase-8-dependent manner (24), therefore we investigated the involvement of SPARC in caspase- 8 activation in our experimental model. First, we analysed the activation of this protease after SPARC silencing. As shown in Fig. 3A, the bands corresponding to the active cleaved forms of caspase- 8 observed in WIN/TRAILtreated cells were almost invisible in silenced cells.

Then, we assessed the possible interaction between SPARC and caspase- 8 in MG63 cells by co-immunoprecipitation studies using a caspase- 8 antibody (against an epitope to C-terminal region) that recognizes both the full length and the cleaved forms of the protein. As shown in Fig. 3B, caspase-8 


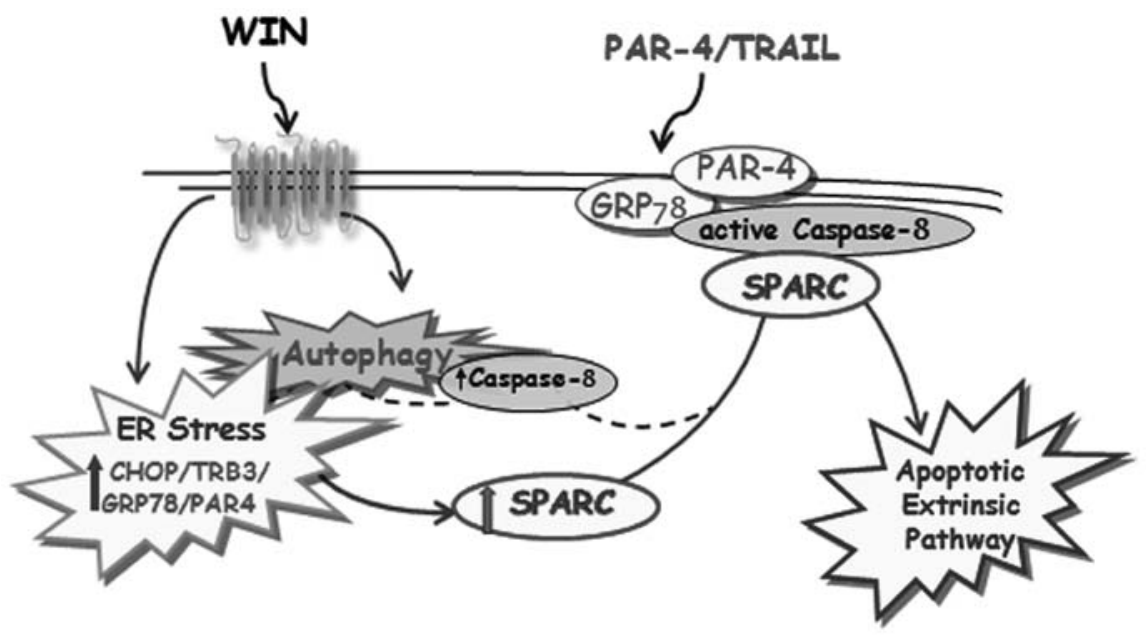

Figure 5. Illustration of the putative pathway induced by WIN to sensitize osteosarcoma cells to TRAIL-dependent apoptosis.

and SPARC co-immunoprecipitated in a reciprocal fashion after WIN treatment. Instead, this interaction disappeared when the cells were treated with WIN/TRAIL combination, demonstrating that the cleavage of caspase- 8 induced by the combined treatment prevents the SPARC immunoprecipitation.

The SPARC increase is related to WIN-induced ER stress. Based on the previously demonstrated role of WIN-induced ER stress and autophagic process on MG63 cell viability (21), we hypothesized a possible relationship between ER stress induction and the SPARC level. Recently, it has been reported that the overexpression of SPARC induces apoptosis in neuroblastoma cells mediated by the induction of ER stress (25). To establish whether a similar relationship occurs in our experimental system, we analysed the level of CHOP, a marker of ER stress, in WIN-treated SPARC silenced cells. As shown in Fig. 4A, the increase in the level of CHOP, observed after WIN-treatment, was not modified by the reduced SPARC expression in silenced cells. Notably, we demonstrated that SPARC increase was a consequence of the activation of the transcriptional factor CHOP induced by WIN. In fact, CHOP silencing which almost completely suppressed the basal levels of CHOP, markedly reduced WIN-induced SPARC increase (Fig. 4B) while the downregulation of GRP78/Bip, another ER stress marker, whose levels are positively regulated by the cannabinoid, did not modify SPARC upregulation induced by WIN treatment (Fig. 4C).

\section{Discussion}

The importance to know the expression protein profile of tumor cells has been widely demonstrated as the response of cells to antineoplastic drugs strongly depends on its specific molecular pattern. It is now evident that a protein can act as an oncogene in a specific tumor model, but as a tumor suppressor in another one and, consequently, its induced downregulation or upregulation can lead to different cell responses. An example of this assumption is SPARC with its different tissuespecific behaviour, which has been examined in a variety of cancer cells.
In this study we analysed the role of SPARC in osteosarcoma cells and, in particular, its involvement in WIN/TRAILinduced cell death. In MG63 cells, although WIN is not able to induce cell death, it sensitizes them to apoptosis induced by WIN/TRAIL combined treatment (21). Our present data strongly indicate for the first time that the synthetic cannabinoid WIN induced a clear increase in the level of SPARC which remained high also in cells treated with WIN/TRAIL combination. Silencing of SPARC counteracted WIN/TRAILinduced cell death as verified through both morphological and cytotoxicity assays. Thus, we conclude that in all likelihood in this tumor model SPARC behaves as a tumor suppressor factor.

In several cell death mechanisms, the role exerted by the activation of caspase- 8 , a canonical membrane upstream protease, in sensitizing cancer cells to apoptosis induced by TRAIL has been demonstrated. In a previous study we demonstrated the activation of caspase- 8 in osteosarcoma cells as a consequence of a marked increase in PAR-4 level after WIN or WIN/TRAIL combined treatment and a concomitant translocation of GRP78, a marker of ER stress, to cell surface, thus correlating ER-stress induction and the activation of extrinsic apoptotic pathway (21). In this study we identified further components of membrane microdomain responsible for the cytotoxic effect of WIN/TRAIL treatment. In fact, in our experimental model, similarly to that observed by Tang and Tai (24) in colorectal cancer, the activation of caspase- 8 seemed to be related with the interaction with SPARC as demonstrated by us through co-immunoprecipitation analysis. The interaction was abrogated after WIN/TRAIL treatment owing to the cleavage and activation of caspase-8.

The relationship between SPARC increase and WINdependent ER stress induction was also particularly interesting. To our knowledge only one study reports that SPARC overexpression triggered ER-stress and thereby unfolded protein response (UPR) in neuroblastoma cells (25). In our experimental conditions the reduction in SPARC level did not modify the WIN-dependent increase in the level of CHOP, a main transcription factor mediator of the cell response to ER-stress. Instead, CHOP downregulation was accompanied 
by the reduction in SPARC level, thus, indicating an inverse relationship between ER-stress induction and SPARC increase.

Overall, data reported in the present study, and summarized in Fig. 5, demonstrate for the first time that in osteosarcoma cells WIN induces a marked increase in the level of SPARC and that in these cells SPARC cooperates for the sensitization to TRAIL action by modulating the translocation of caspase- 8 in the plasma membrane. Caspase- 8 forms a microdomain together with PAR-4 and GRP-78 which, acting as TRAIL receptor, induce caspase- 8 cleavage after the addition of the cytokine with the consequent triggering of the extrinsic apoptotic pathway.

\section{References}

1. Ottaviani G and Jaffe N: The epidemiology of osteosarcoma. Cancer Treat Res 152: 3-13, 2009.

2. Letourneau PA, Xiao L, Harting MT, Lally KP, Cox CS Jr, Andrassy RJ and Hayes-Jordan AA: Location of pulmonary metastasis in pediatric osteosarcoma is predictive of outcome. J Pediatr Surg 46: 1333-1337, 2011.

3. Brekken RA and Sage EH: Sparc a matricellular protein: A crossroad of cell-matrix communication. Med Sci Monit 13: $25-30,2001$

4. Lane TF and Sage EH: The biology of SPARC, a protein that modulates cell-matrix interactions. FASEB J 8: 163-173, 1994.

5. Motamed K, Funk SE, Koyama H, Ross R, Raines EW and Sage EH: Inhibition of PDGF-stimulated and matrix-mediated proliferation of human vascular smooth muscle cells by SPARC is independent of changes in cell shape or cyclin-dependent kinase inhibitors. J Cell Biochem 84: 759-771, 2002.

6. Delany AM, Kalajzic I, Bradshaw AD, Sage EH and Canalis E: Osteonectin-null mutation compromises osteoblast formation, maturation, and survival. Endocrinology 144: 2588-2596, 2003.

7. Alford AI and Hankenson KD: Matricellular proteins: Extracellular modulators of bone development, remodeling, and regeneration. Bone 38: 749-757, 2006.

8. Kunigal S, Gondi CS, Gujrati M, Lakka SS, Dinh DH, Olivero WC and Rao JS: SPARC-induced migration of glioblastoma cell lines via uPA-uPAR signaling and activation of small GTPase RhoA Int J Oncol 29: 1349-1357, 2006.

9. Rentz TJ, Poobalarahi F, Bornstein P, Sage EH and Bradshaw AD: SPARC regulates processing of procollagen I and collagen fibrillogenesis in dermal fibroblasts. J Biol Chem 282: 22062-22071, 2007.

10. Tai IT and Tang MJ: SPARC in cancer biology: Its role in cancer progression and potential for therapy. Drug Resist Updat 11: 231-246, 2008.

11. Bradshaw AD and Sage EH: SPARC, a matricellular protein that functions in cellular differentiation and tissue response to injury. J Clin Invest 107: 1049-1054, 2001.

12. Bellahcène A and Castronovo V: Increased expression of osteonectin and osteopontin, two bone matrix proteins, in human breast cancer. Am J Pathol 146: 95-100, 1995.
13. Horie K, Tsuchihara M and Nakatsura T: Silencing of secreted protein acidic and rich in cysteine inhibits the growth of human melanoma cells with $\mathrm{G}$ arrest induction. Cancer Sci 101: 913-919, 2010.

14. Yunker CK, Golembieski W, Lemke N, Schultz CR, Cazacu S, Brodie C and Rempel SA: SPARC-induced increase in glioma matrix and decrease in vascularity are associated with reduced VEGF expression and secretion. Int J Cancer 122: 2735-2743, 2008.

15. Shi Q, Bao S, Maxwell JA, Reese ED, Friedman HS, Bigner DD, Wang XF and Rich JN: Secreted protein acidic, rich in cysteine (SPARC), mediates cellular survival of gliomas through AKT activation. J Biol Chem 279: 52200-52209, 2004.

16. Mateo F, Meca-Cortés $\mathrm{O}$, Celià-Terrassa $\mathrm{T}$, Fernández $\mathrm{Y}$, Abasolo I, Sánchez-Cid L, Bermudo R, Sagasta A, RodríguezCarunchio L, Pons M, et al: SPARC mediates metastatic cooperation between CSC and non-CSC prostate cancer cell subpopulations. Mol Cancer 13: 237, 2014.

17. Cheetham S, Tang MJ, Mesak F, Kennecke H, Owen D and Tai IT: SPARC promoter hypermethylation in colorectal cancers can be reversed by 5-Aza-2'deoxycytidine to increase SPARC expression and improve therapy response. Br J Cancer 98: 1810-1819, 2008.

18. Chen ZY, Zhang JL, Yao HX, Wang PY, Zhu J, Wang W, Wang X, Wan YL, Chen SW, Chen GW, et al: Aberrant methylation of the SPARC gene promoter and its clinical implication in gastric cancer. Sci Rep 4: 7035, 2014.

19. Mok SC, Chan WY, Wong KK, Muto MG and Berkowitz RS: SPARC, an extracellular matrix protein with tumor-suppressing activity in human ovarian epithelial cells. Oncogene 12 : 1895-1901, 1996.

20. Zhang JL, Chen GW, Liu YC, Wang PY, Wang X, Wan YL, Zhu J, Gao HQ, Yin J, Wang W, et al: Secreted protein acidic and rich in cysteine (SPARC) suppresses angiogenesis by down-regulating the expression of VEGF and MMP-7 in gastric cancer. PLoS One 7: e44618, 2012

21. Notaro A, Sabella S, Pellerito O, Di Fiore R, De Blasio A, Vento R, Calvaruso G and Giuliano M: Involvement of PAR-4 in cannabinoid-dependent sensitization of osteosarcoma cells to TRAIL-induced apoptosis. Int J Biol Sci 10: 466-478, 2014.

22. Pellerito O, Notaro A, Sabella S, De Blasio A, Vento R, Calvaruso G and Giuliano M: WIN induces apoptotic cell death in human colon cancer cells through a block of autophagic flux dependent on PPAR $\gamma$ down-regulation. Apoptosis 19: 1029-1042, 2014.

23. Portanova P, Notaro A, Pellerito O, Sabella S, Giuliano M and Calvaruso G: Notch inhibition restores TRAIL-mediated apoptosis via AP1-dependent upregulation of DR4 and DR5 TRAIL receptors in MDA-MB-231 breast cancer cells. Int J Oncol 43: 121-130, 2013.

24. Tang MJ and Tai IT: A novel interaction between procaspase 8 and SPARC enhances apoptosis and potentiates chemotherapy sensitivity in colorectal cancers. J Biol Chem 282: 34457-34467, 2007.

25. Sailaja GS, Bhoopathi P, Gorantla B, Chetty C, Gogineni VR, Velpula KK, Gondi CS and Rao JS: The secreted protein acidic and rich in cysteine (SPARC) induces endoplasmic reticulum stress leading to autophagy-mediated apoptosis in neuroblastoma. Int J Oncol 42: 188-196, 2013. 